\title{
Bioavailability of freeze-dried and spray-dried grapefruit juice vitamin $\mathbf{C}$
}

\section{Camacho, M.M. ${ }^{\text {; }}$ Igual, M. ${ }^{\text {; }}$ Martínez-Lahuerta, J.J. ${ }^{\text {; }}$ Martínez-Navarrete, $\mathbf{N}^{\text {a* }}$}

${ }^{a}$ CUINA group. Department of Food Technology. Universitat Politècnica de València, Valencia, Spain

${ }^{\text {b }}$ Centre Auxiliar Juan Llorens, Valencia (Spain).

*E-mail of the corresponding author: nmartin@tal.upv.es

\begin{abstract}
An alternative as to offer higher stable and easy handling than fresh fruit is in powdered form, as long as the process used to obtain it ensures a high quality product. The objective of this study was to compare the bioavailability of the vitamin $C$ of a juice prepared from powdered grapefruit obtained by freeze-drying and by spray-drying. A trial was conducted with 11 healthy volunteers. A relative increase of 1,4-25,8\% of blood serum vitamin $C$ concentration was quantified after juices intake, with no significant differences $(p>0.05)$ due to the process used to obtain the powder.
\end{abstract}

Keywords: vitamin C; bioavailability in humans; grapefruit powder; freezedrying; spray-drying. 


\section{Introduction}

Consumer attention is currently focused on healthy eating habits. Fruit juices can be clearly included in a healthy diet as they contain different health-promoting bioactive compounds, mainly vitamin $\mathrm{C}$ and phenolic. Nevertheless, consumer also looks for high quality, stable and ease to handle foods. In this sense, fruit powder may be an interesting alternative to offer a juice after being rehydrated. Different techniques can be used to obtain food powder, freeze-drying and spray-drying being the most commonly used as they offer very high quality products. A lot of studies, too much to be cited in the context of this manuscript, have been carried out to evaluate the impact of different food processes on the product quality. In fact, many processes have been optimized taking into account these quality aspects. Nevertheless, the studied quality attributes are related to the composition, physical, chemical or biochemical properties or sensory aspects, among others. Nonetheless, we have not found any study investigating the impact of the processes on the bioavailability of the bioactive compounds potentially responsible for the functional value of foods.

The biological functions of vitamin $\mathrm{C}$ depend upon its ability to act as an electron donor. Among these it acts as cofactor for a variety of enzymes with critical functions throughout the body, functions as a highly effective water-soluble antioxidant and it is suggested that is involved in the regeneration of vitamin $\mathrm{E}$ in vivo ${ }^{[1,2]}$. Reported beneficial effects of this vitamin include increased baroreflex sensitivity, improved endothelial vascular function, augmented inotropic and thermogenic response to beta-adrenergic stimulation, decreased systemic inflammation and reduced fluid requirements during recovery from thermal injury, improved fatigue resistance, increased iron bioavailabilty and decreased risk of cardiovascular diseases ${ }^{[3,4,5]}$. Severe vitamin $\mathrm{C}$ deficiency produces scurvy. Despite humans are unable to synthesize their own vitamin $\mathrm{C}$, it must be easily obtained from the diet, principally through a adequate fruit and vegetable consumption ${ }^{[1]}$.

The research on which this study is part, started with the comparison of freeze drying (FD) and spray drying (SD) as two drying technologies with which to obtain grapefruit powder with the lowest water content, hygroscopicity, luminosity, color change and the highest content of total phenolic, total carotenoids, vitamin $\mathrm{C}$ and antioxidant capacity, despite the highest powder yield ${ }^{[6]}$. In that study, response surface methodology (RSM) was used to evaluate the effect of three process variables on the different mentioned properties. The considered process variables were the amount of gum Arabic and bamboo fiber added to prevent the powder stickiness, also as the feed inlet water content or the inlet air temperature in the case of FD and SD processes, respectively. The conclusion of that study was that FD might be proposed as a better technology than SD with which to obtain grapefruit powder with a higher vitamin $\mathrm{C}$ and total carotenoids content. Taking these 
results into account, the aim of the present study was to investigate if there was a different impact of the previously optimized FD and SD processes on the bioavailability of the vitamin C.

\section{Materials and Methods}

\subsection{Tested grapefruit products}

The volunteers who participated in the trial consumed two grapefruit juices. They were obtained by rehydration of the grapefruit powder obtained by freeze-drying (FDJ) and by spray-drying (SDJ) of a previously formulated grapefruit mix. The same grapefruit (Citrus paradise var. Star Ruby) batch, purchased in a local supermarket (Valencia, Spain), was used to obtain both powdered products which were processed taking into account the optimum conditions proposed in the previously mentioned study ${ }^{[6]}$. Briefly, peeled and grounded grapefruit was mixed with $4.2 \mathrm{~g} \mathrm{GA}+0.6 \mathrm{~g} \mathrm{BF} / 100$ grapefruit pulp to obtain FDJ or with $4.3 \mathrm{~g} \mathrm{GA}+2.1 \mathrm{~g} \mathrm{BF} / 100$ liquidized grapefruit to obtain SDJ. No shelf temperature was applied during FD and inlet air temperature for SD was $120^{\circ} \mathrm{C}$. FD cake was crushed to obtain a powder which was vacuum packed and freezing stored until used. SD powder was stored in the same conditions. The vitamin $\mathrm{C}$ content of the grapefruit batch used in the study, analyzed as described below, was $41.8 \mathrm{mg} / 100 \mathrm{~g}$ pulp.

Each powder was rehydrated in the day of the trial, two hours before being given to the participants. The amount of water to be added to the powder was calculated in order to ensure a juice which offers the same amount of grapefruit own's solutes present in the grapefruit batch used for the study. The corresponding mass balance was applied to this end, taking into account the water mass fraction of the grapefruit, the amount of GA and BF added to the prepared mix and the water mass fraction of the obtained powders.

\subsection{Research participants}

Ethical approval for the trial was obtained from the Ethics Committee of the Universitat Politècnica de València (Valencia, Spain). The trial was conducted with a total of 11 healthy volunteers from different countries of South America and Europe, who met the inclusion and exclusion criteria established for the study. Inclusion criteria included age within the range of 18-40 years, with a normal body mass index (BMI between 19 and 29 $\mathrm{kg} / \mathrm{m}^{2}$ ). Exclusion criteria included smokers and people with special dietary habits (vegetarians, vegans, macrobiotics, etc.), allergic to any food, pregnant or with intention to be in the trial period, medicated (includes dietary supplements: vitamin, protein, etc.), 
suffering infectious diseases by blood (positive serology for hepatitis B virus and $\mathrm{C}$ or human immunodeficiency virus), presenting some picture of intestinal malabsorption, suffering metabolic diseases (diabetes, dyslipidemia, thyroid disorders, etc.) and suffering cardiovascular or kidney diseases (high blood pressure, renal insufficiency, etc.). All the 11 selected participants signed the corresponding informed consent after being explained about the nature, purpose and risks of the study.

\subsection{Experimental design and blood sample collection}

A crossover design was considered for the clinical trial. With this design, each research participant consumed both juices, obtained from FD and SD rehydrated powders, in different time periods separated by 15 days. One of the advantages of crossover designs in contrast to parallel ones is that the former could yield the same level of statistical power or precision with a lower sample size, as each subject serves as his/her own matched control, thus blocking the variability due to the subjects.

All the participants in the study were cited on two different days, one for FDJ consumption and another one for SDJ. Each day, each subject underwent a first overnight fasting blood test and another one $4 \mathrm{~h}$ after having drunk $400 \mathrm{~g}$ of the corresponding juice which was given with no meal. They were not allowed to eat anything in the $4 \mathrm{~h}$ between extractions. Two blood samples from each participant were collected into BDVacutainer SSTII Advance Tubes (REF 367953), that were allowed to coagulate for $30 \mathrm{~min}$ and after centrifuged at $20^{\circ} \mathrm{C}$ and $1500 \mathrm{x} g$ for $10 \mathrm{~min}$ to separate the serum which was analyzed in its vitamin $\mathrm{C}$ content. Serum concentration of ascorbic and dehydroascorbic acids, as related to grapefruit juice vitamin $\mathrm{C}$ bioavailability, was analyzed by means of HPLC as described below. The mean value (and standard deviation) of the two tubes of each subject was considered as the corresponding result.

\subsection{Vitamin C analysis}

The total vitamin $\mathrm{C}$ content of the grapefruit batch used for the study was analyzed as described by Igual et al. ${ }^{[7]}$ and $\mathrm{Xu}$ et al. ${ }^{[8]}$. The same methodology slightly modified was used to quantify the total vitamin $\mathrm{C}$ content present in serum. Briefly $0.5 \mathrm{~mL}$ serum was mixed with $1 \mathrm{~mL}$ of a $20 \mathrm{~g} / \mathrm{L}$ DL-dithiothreitol solution for $2 \mathrm{~h}$ at room temperature and in darkness. Afterwards, $1 \mathrm{~mL}$ of this mixture was extracted with $2.25 \mathrm{~mL} 0.1 \%$ oxalic acid for $3 \mathrm{~min}$ and immediately filtered through a $0.45 \mathrm{~mm}$ membrane filter before injection. The HPLC conditions were: Ultrabase-C18, $5 \mathrm{~mm}(4.6$ x $250 \mathrm{~mm})$ column (Análisis Vínicos, Spain); mobile phase $0.1 \%$ oxalic acid, volume injection $20 \mathrm{~mL}$, flow rate $1 \mathrm{~mL} / \mathrm{min}$, detection at $243 \mathrm{~nm}$ and at $25^{\circ} \mathrm{C}$. A standard solution (Panreac, Spain) was prepared. 


\subsection{Statistical analysis}

The difference in serum vitamin $\mathrm{C}$ concentration before and after the juices intake was considered as the response variable, taken as a measurement of the juice vitamin $\mathrm{C}$ bioavailabity. A multifactor analysis of variance was performed for this response variable taking into account the factors individual and juice, with a total of 11 individuals and 2 juices tested. Statgraphics Centurion XVI.II for Windows was used for the statisticall analysis.

\section{Results and discussion}

Figure 1 shows the vitamin $\mathrm{C}$ concentration found in blood serum. The fasting serum vitamin $C$ values of the paticipants in the trial varied between 10.1 and $13.6 \mathrm{mg} / \mathrm{L}$. As expected from the country of habitual residence of the participants, all of them were above the adequate level of plasma vitamin $C$ (i.e. $50 \mu \mathrm{mol} / \mathrm{L}^{[1}=8.80 \mathrm{mg} / \mathrm{L}$ ), with any hypovitaminosis case detected.

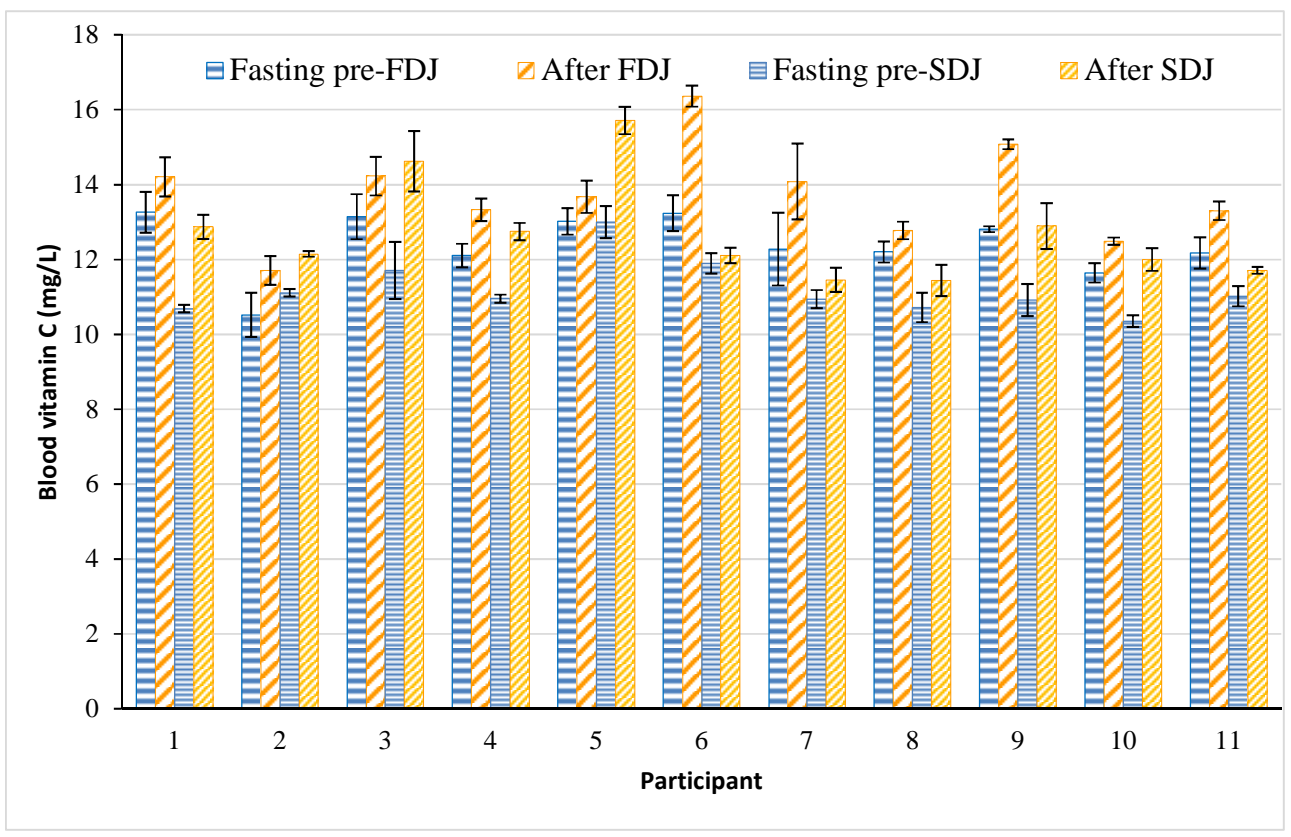

Fig. 1. Mean value and standard deviation of serum blood vitamin $C$ concentration analyzed in the participants the days of consuming reconstituted freeze-dried (FDJ) and spray-dried (SDJ) juices, both fasting and $4 \mathrm{~h}$ after the intake of each juice. 
The level of vitamin $\mathrm{C}$ in the blood serum of all the participants in the trial increased after the ingestion of each juice, the level reached varing between 11.1 and $16.6 \mathrm{mg} / \mathrm{L}$ (Fig. 1). This increase, compared to the basal fasting level, evidenced the absorption of vitamin $\mathrm{C}$ from juices. When comparing the circulating concentration of vitamin $\mathrm{C}$ fasting with the one $4 \mathrm{~h}$ following the ingestion of the juices, an increase between 0.17 and $3.27 \mathrm{mg} / \mathrm{L}$ was observed. The increase related to the fasting level, for each participant, is shown in Figure 2 , this relative increase being in the range $1.4-25.8 \%$.

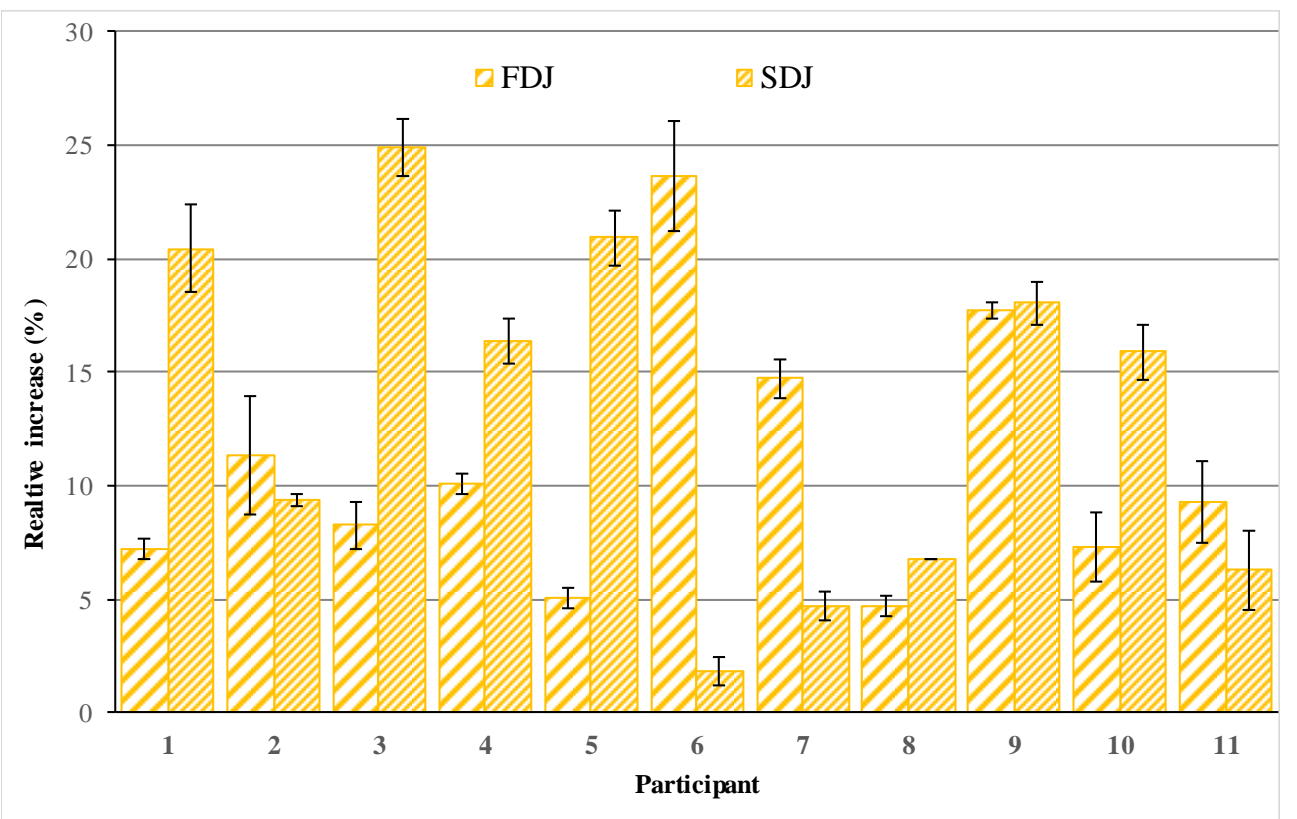

Fig. 2. Mean value and standard deviation of the relative increase of serum blood vitamin $C$ concentration suffered by the participants due the intake of reconstituted freeze-dried (FDJ) and spray-dried (SDJ) juices.

Taking into account the average values of the 22 relative increase values considered, for each of the 11 people on the day of the intake of each of the juices, only three times the relative increase in the concentration of vitamin $\mathrm{C}$ in blood compared to fasting baseline was below 5\%. In eight cases this increase was between 5 and $10 \%$, in three between 10 and $15 \%$, in four between 15 and $20 \%$ and in four between 20 and $25 \%$. In order to compare the blood vitamin $\mathrm{C}$ increase caused in each participant by FDJ and SDJ ingestion, an ANOVA was carried out with the factors juice and individual. No significant differences ( $>0.05$ ) were observed in any case. In this way, the mean $10.85 \%$ relative increase of blood circulating vitamin $\mathrm{C}$ in the participants in the trial caused by FDJ ingestion was not 
significant different $(\mathrm{p}>0.05)$ to the mean $13.23 \%$ detected after SDJ ingestion (standard error 2.5). As far as the individuals is concerned, the mean relative increase caused by the two juices tested was not significant different $(\mathrm{p}>0.05)$ in any of them, the lowest relative incrase being $5.7 \%$ and the highest $17.9 \%$ (standard error 0.06). In this way a relative increase of vitamin $\mathrm{C}$ in blood due to juices intake of $12 \%$ can be assumed as a grand mean of the 22 average values considered.

\section{Conclusions}

Vitamin $\mathrm{C}$ present in juices prepared by rehydration of grapefruit powder obtained by freeze-drying and by spray-drying is absorbed by the human body, without differences in its bioavailability due to the different process applied to obtain the powder.

\section{Acknowledgements}

The authors thank the Ministerio de Economía y Competitividad for the financial support given through the Project AGL 2012-39103.

\section{References}

[1] Pearson, J.F.; Pullar, J.M.; Wilson, R.; Spittlehouse, J.K.; Vissers, M.C.M.; Skidmore, P.M.L.; Willis, J.; Cameron, V.A.; Carr, A.C. Vitamin C Status Correlates with Markers of Metabolic and Cognitive Health in 50-Year-Olds: Findings of the CHALICE Cohort Study. Nutrients, 2017, 9, 831.

[2] Bruno, R.S.; Leonard, S.W.; Atkinson, J.; Montined, T.J.; Ramakrishnane, R.; Bray, T.M.; Trabera, M.G. Faster plasma vitamin E disappearance in smokers is normalized by vitamin C supplementation. Free Radical Biology and Medicine, 2006, 40(4), 689697.

[3] Davis, J.L.; Paris, H.L.; Beals, J.W.; Binns, S.E.; Giordano, G.R.; Scalzo, R.L.; Schweder, M.M.; Blair, E.; Bell, C. Liposomal-encapsulated Ascorbic Acid: Influence on Vitamin C Bioavailability and Capacity to Protect Against Ischemia-Reperfusion Injury. Nutrition and Metabolic Insights, 2016, 9, 25-30.

[4] Monahan, K.D.; Eskurza, I.; Seals, D.R. Ascorbic acid increases cardiovagal baroreflex sensitivity in healthy older men.American Journal of Physiology-Heart and Circulatory Physiology, 2004, 286(6), H2113-7.

[5] Ye, Z.; Song, H. Antioxidant vitamins intake and the risk of coronary heart disease: meta-analysis of cohort studies. European Journal of Preventive Cardiology, 2008,15(1),26-34. 
[6] Agudelo C., Igual M., Camacho M.M.; Martínez-Navarrete N. Effect of process technology on the nutritional, functional, and physical quality of grapefruit powder. Food Science Technology International, 2017, 23(1), 61-74.

[7] Igual, M.; Ramires, S.; Mosquera, L.H.; Martínez-Navarrete, N. Optimization of spray drying conditions for lulo (Solanum quitoense L.) pulp. Powder Technology, 2014, 256, 233-23.

[8] Xu, G.; Liu, D.; Chen, J.; Ye, X.; Maa, Y.; Shi, J. Juice components and antioxidant capacity of citrus varieties cultivated in China. Food Chemistry, 2008, 106, 545-551. 